\title{
O CONTROLE DO USO DE ANIMAIS PARA ENSINO E PESQUISA
}

\author{
Vanessa Carli Bones ${ }^{1}$, Elaine Cristina de Oliveira Sans ${ }^{1}$, \\ Ricardo Alexandre Franco Simon ${ }^{2}$, Carla Forte Maiolino Molento ${ }^{1}$ \\ 1 Universidade Federal do Paraná - vcb.vete@gmail.com \\ 2 Conselho Regional de Medicina Veterinária do Paraná
}

RESUMO: A regulamentação da experimentação animal está fundamentada na preocupação ética de não se infringir sofrimento aos animais, demonstrada pelos movimentos sociais de proteção animal, pela legislação abordando o tema, pelas Comissões de Ética no Uso de Animais, entre outros. O objetivo desta revisão foi estudar o controle do uso de animais para ensino e pesquisa em alguns países, escolhidos aleatoriamente, comparando-os com a situação brasileira e paranaense. $O$ controle é feito por diferentes instituições em diferentes países, a saber: pelo Presidente e Parlamento na África do Sul; instituição autônoma no Canadá; Departamento da Agricultura nos Estados Unidos; Ministério da Educação, Cultura, Esportes, Ciência e Tecnologia no Japão; Ministério Federal de Alimentos, Agricultura e Proteção do Consumidor na Alemanha; Ministério da Agricultura na França e Suécia; Ministério da Saúde, Bem-estar e Esporte na Holanda; Home Office no Reino Unido; Conselho Federal na Suíça; e Conselho Nacional de Saúde e Pesquisa Médica na Austrália. No Brasil o controle é feito pelo Ministério da Ciência e Tecnologia, havendo duas Leis Federais, um Decreto e uma Resolução; no Paraná, há o Código Estadual de Proteção aos Animais. O conhecimento do trabalho de instituições responsáveis pelo controle da experimentação animal em diferentes países pode auxiliar o aprimoramento deste processo no Brasil. Tal controle é urgente pela necessidade de proteção do bem-estar animal e pela preocupação crescente da sociedade.

Palavras-chave: aulas práticas com animais; ética; experimentação; legislação; regulamentação

\section{ANIMAL USE CONTROL IN TEACHING AND RESEARCH}

\begin{abstract}
The animal experimentation regulation is based on the ethical concern regarding not to cause animal suffering, expressed by social movements in favour of the animal protection, legislation on this subject, Animal Use Ethics Committees, amongst others. The objective of this review was to study the control of animal use in teaching and research in some countries, randomly selected, comparing them to the situation in Brazil and the State of Paraná. The control process is performed by different institutions in different countries: the President and Parliament in South Africa; autonomous institution in Canada; Department of Agriculture in United States; Ministry of Education, Culture, Sports, Science and Technology in Japan; Federal Ministry of Food, Agriculture and Consumer Protection in Germany; Ministry of Agriculture in France and Sweden; Ministry of Health, Welfare and Sport in Netherlands; Home Office in United Kingdom; Federal Council in Switzerland; and National Health and Medical Research Council in Australia. The control is responsibility of the Ministry of Science and Technology in Brazil, under two Federal Laws, a Decree and a Resolution; in the State of Paraná, there is the State Animal Protection Code. Knowing the work of institutions responsible for the control of the animal experimentation in different countries may help the improvement of this process in Brazil. Such control is urgent due to the need for animal welfare protection and the increasing ethical concern from society in general.
\end{abstract}

Key words: Animals in practical lessons; ethics; experimentation; legislation; regulation 


\section{INTRODUÇÃO}

A regulamentação do uso de animais em ensino e pesquisa está fundamentada na preocupação em não se infringir sofrimento. De acordo com Paixão (2008), o surgimento de leis em diversos países, o aparecimento das Comissões de Ética no Uso de AnimaisCEUAs, o controle por agências de financiamento e novas políticas editoriais são exemplos de fenômenos associados ao controle do uso de animais para pesquisa. As sociedades ocidentais, que haviam delegado tais decisões éticas aos profissionais da área, parecem buscar uma reorganização do assunto (Benson e Rollin, 2004). Tal reorganização vem acontecendo principalmente por meio do aumento de regulamentação, inclusive com surgimento de leis de cunho proibitivo e prescritivo.

Um exemplo do início do processo de controle do uso de animais e da preocupação ética envolvida está relacionado com a emergência de duas correntes em favor dos animais no final da segunda guerra mundial. A primeira relaciona-se aos animais usados em pesquisas biomédicas e a outra aos animais usados em agricultura. De 1900 até 1920, o número de animais usados em pesquisas foi baixo e constante. Depois de 1920 os números aumentaram, quando grandes quantidades de dinheiro foram gastas em pesquisas e produção de drogas (Rollin, 2003). Também a atuação das sociedades protetoras de animais foi importante para o processo de controle do uso de animais no mundo. A primeira delas surgiu na Inglaterra em 1824 e atualmente se denomina Sociedade Real para a Prevenção da Crueldade contra Animais (The Royal Society for the Prevention of Cruelty to AnimalsRSPCA) (RSPCA, 2008). Na França, a primeira sociedade para a proteção dos animais foi criada em 1845 e em anos posteriores surgiram entidades semelhantes na Alemanha, Bélgica, Áustria, Holanda e Estados Unidos (Raymundo e Goldim, 2002).

De maneira clara, a preocupação da sociedade com o bem-estar animal e a experimentação é crescente. No Brasil este fato é evidenciado pela publicação de livros e outros trabalhos relacionados (Greif e Tréz, 2000; Dias, 2000; Levai, 2004; Rodrigues, 2006; Naconecy, 2007). Desta forma, existe impulso da sociedade para a evolução do sistema de controle do uso de animais para ensino e pesquisa no país.

Conhecer diferentes sistemas de controle da experimentação animal, observar aspectos presentes nas leis de outros países e acompanhar o debate internacional são ações fundamentais para que se possa avançar no campo da ética, do bem-estar animal e de uma ciência de qualidade (Paixão, 2007). Verificam-se provisões legais que regulamentam o uso de animais em ensino e pesquisa em países como Alemanha, Austrália, Áustria, Bermuda, Brasil, Canadá, China, Coréia, Costa Rica, Croácia, Eslovênia, Estados Unidos, Filipinas, Finlândia, França, Grécia, Holanda, Ilhas Salomão, Índia, Irlanda, Itália, Japão, Noruega, Nova Zelândia, Peru, Polônia, Portugal, República Checa, República das Ilhas Fiji, Romênia, Rússia, Suécia, Suíça e Taiuã (FRAME, 2008). Adicionalmente, a maioria dos países africanos possui leis de bem-estar animal (Aceme, 2009). Esta revisão tem por objetivo apresentar as formas de controle do uso de animais em ensino e pesquisa de onze países, localizados em cinco continentes e selecionados aleatoriamente, enriquecendo as possibilidades a serem consideradas para o aprimoramento do sistema regulamentador no Brasil e particularmente no Paraná. 


\section{As comissões de ética no uso de animais- CEUAs}

Assim como a legislação vigente, as CEUAs contribuem para o controle do uso de animais em ensino e pesquisa. Segundo Paixão (2004), um dos países pioneiros a organizar tais comissões foi a Suécia, cuja obrigatoriedade data de 1979. Sua missão era avaliar experimentos em relação à dor ou sofrimento aos animais. Com a revisão da Lei de Proteção Animal daquele país (Animal Protection Act- 1977) em 1988, os experimentos passaram a ter exigência de aprovação por uma CEUA antes de serem iniciados. Nos Estados Unidos, a revisão dos protocolos de pesquisa por CEUAs tornou-se obrigatória em 1985, em um dos processos de revisão da lei americana de bem-estar animal (Animal Welfare Act - 1966), após o episódio de Silver Spring. Tal episódio é descrito detalhadamente por Rudacille (2001).

Segundo Paixão (2004), pode-se dizer que o foco de atuação de uma CEUA é o bem-estar animal e a avaliação do mérito científico da pesquisa, buscando o cumprimento da política dos 3Rs (Russell e Burch, 1992), que significa Substituição, Redução e Refinamento nas pesquisas com animais. De acordo com Shapiro (2000), além de garantir tratamento ético aos animais usados para propósitos científicos, as CEUAs devem fazer uma análise utilitarista dos benefícios de um projeto em relação aos seus custos em termos de sofrimento animal. Assim, as CEUAs não podem restringir seus trabalhos à averiguação de condições de manutenção e da qualidade técnica dos procedimentos realizados, mas acima de tudo questionar se um procedimento deve ser realizado ou não. Em se tratando da composição das CEUAs, diferentes países levam em conta categorias distintas de membros, mas de forma geral podem ser identificados representantes da ciência (professores ou cientistas), médicos veterinários e representantes da sociedade comprometidos com a proteção animal (Middleton, 2005).

De acordo com Chaves (2000), 14 instituições brasileiras possuíam CEUAs na época. Os princípios éticos adotados pelas CEUAs não puderam ser avaliados pela dificuldade de obtenção de dados visto que as Comissões ainda trabalham de forma isolada. Em 2008 foram identificadas 32 CEUAs; porém, várias comissões conhecidas não foram incluídas, como as CEUAs pertencentes às universidades estaduais paulistas Unesp, Unicamp e USP (Autor Desconhecido, 2008). Percebe-se dificuldade em termos de obtenção de informações e de coerência nas formas de atuação das CEUAs.

Visando suprir a necessidade urgente do controle do uso de animais em ensino e pesquisa, em fevereiro de 2008 o Conselho Federal de Medicina Veterinária publicou a Resolução 879 (CFMV, 2008). Tal resolução visa disciplinar, uniformizar e normatizar o uso científico de animais sencientes no ensino e na pesquisa médica-veterinária e zootécnica em nível nacional, através da criação de comissões de ética no uso de animais nas instituições, do zelo pelo bem-estar animal, entre outras medidas.

De forma semelhante, em outubro de 2008 foi aprovada a Lei Federal no 11.794 (Brasil, 2008b), sendo em julho de 2009 aprovado o Decreto no 6.899 (Brasil, 2009), que regulamenta a referida lei. Em suma, a Lei regulamenta a criação e a utilização de animais em atividades de ensino e pesquisa científica no Brasil. Ela determina, entre outras providências, a criação do Conselho Nacional de Controle de Experimentação Animal - CONCEA, cuja competência envolve 0 cumprimento das normas relativas à utilização humanitária de animais com 
finalidade de experimentação; 0 credenciamento de instituições para criação ou utilização de animais; a avaliação da introdução de técnicas alternativas ao uso de animais, a manutenção de um cadastro dos procedimentos de ensino e pesquisa realizados ou em andamento, assim como dos pesquisadores, a partir de informações remetidas pelas CEUAs. A lei também estabelece a criação de CEUAs em instituições de ensino ou pesquisa com animais, sua composição e responsabilidades; bem como a competência do Ministério da Ciência e Tecnologia no licenciamento das atividades destinadas à criação de animais, ao ensino e à pesquisa científica de que trata esta Lei.

Tais publicações oficiais indicam a presença de provisões para regulamentação do uso de animais em ensino e pesquisa no Brasil, incluindo a existência de CEUAs em instituições que conduzem experimentação. No Estado do Paraná, a criação de CEUAs também é prevista por legislação estadual. O Código Estadual de Proteção aos Animais do Paraná prevê a criação de CEUAs em locais nos quais ocorra vivissecção, composta por no mínimo, três médicos veterinários (Paraná, 2003).

\section{Controle do uso de animais no mundo}

A existência de formas de controle do uso de animais em ensino e pesquisa em diversos países demonstra a crescente preocupação da sociedade em minimizar o sofrimento animal e em criar um ambiente no qual a ciência possa ser desenvolvida de maneira ética. A escolha dos países a serem estudados foi relativamente aleatória, pois se delineou necessariamente a presença de no mínimo um país de cada continente. Tal escolha se baseou ainda na disponibilidade de informações relativas à forma de controle adotada, presentes em sítios da Internet. Além do Brasil, os países estudados foram África do Sul, Alemanha, Austrália, Canadá, Estados Unidos, França, Holanda, Japão, Reino Unido, Suécia e Suíça. Reitera-se que a escolha de tais países não significa que outros não tenham formas de controle ou mesmo que não tenham informações disponíveis online.

\section{Controle do uso de animais na África}

De acordo com Aceme (2009), apesar de não haver dados empíricos sobre a existência ou adequação de políticas e diretrizes nacionais e ou institucionais no uso de animais em pesquisa na África, a maioria dos países ainda não está no mesmo nível que países desenvolvidos.

Além de pesquisas com animais objetivando a busca da cura para inúmeras doenças humanas, a maioria das universidades na África utiliza animais para ensino em medicina veterinária, zootecnia e estudos biomédicos. Atividades envolvendo ensino e pesquisa são incontáveis, o que reforça a importância de contínuos esforços coletivos para a garantia do bem-estar de animais usados para estes propósitos. Neste sentido, alguns países africanos estão desenvolvendo uma estrutura ética e legal, porém, para a maioria, o domínio público destas informações é o maior desafio a ser vencido (Aceme, 2009).

A maioria dos países africanos possui leis objetivando a promoção do bem-estar dos animais, porém em alguns casos, tais leis parecem incluir o bem-estar de animais em geral sem especificar animais usados para ensino e pesquisa. Vários países estão revisando suas leis para promover a proteção de animais usados para ensino e pesquisa, entre outras categorias (Aceme, 2009). Na África do Sul, por exemplo, diretrizes nacionais no uso de animais em ensino, pesquisa e testes foram desenvolvidas pelo Conselho Sul 
Tabela 1- Sistemas de controle do uso de animais para ensino e pesquisa em países selecionados dos cinco continentes, de acordo com instituições responsáveis e níveis de hierarquia.

\begin{tabular}{|c|c|c|c|c|}
\hline & \multirow{2}{*}{ País } & \multicolumn{3}{|c|}{ Instituições responsáveis } \\
\hline & & Nível 1 & Nível 2 & Nível 3 \\
\hline \multirow[t]{2}{*}{ África } & África do Sul & $\begin{array}{l}\text { Presidente do Estado e } \\
\text { Parlamento }\end{array}$ & $\begin{array}{l}\text { Conselho Sul Africano } \\
\text { de Pesquisa Médica }\end{array}$ & \\
\hline & Canadá & $\begin{array}{l}\text { Conselho Canadense de } \\
\text { Cuidados aos Animais }\end{array}$ & & \\
\hline \multirow[t]{2}{*}{ Américas } & Estados Unidos & $\begin{array}{c}\text { Departamento de } \\
\text { Agricultura dos Estados } \\
\text { Unidos }\end{array}$ & $\begin{array}{l}\text { Serviço de Inspeção em } \\
\text { Saúde de Animais e } \\
\text { Plantas }\end{array}$ & \\
\hline & Brasil & $\begin{array}{c}\text { Ministério da Ciência e } \\
\text { Tecnologia }\end{array}$ & $\begin{array}{l}\text { Conselho Nacional de } \\
\text { Controle de } \\
\text { Experimentacão Animal }\end{array}$ & \\
\hline \multirow[t]{4}{*}{ Ásia } & Japão & $\begin{array}{c}\text { Ministério da Educação, } \\
\text { Cultura, Esportes, Ciência e } \\
\text { Tecnologia }\end{array}$ & Conselho da Ciência & \\
\hline & Alemanha & $\begin{array}{l}\text { Ministério Federal de } \\
\text { Alimentos, Agricultura e } \\
\text { Proteção do Consumidor }\end{array}$ & $\begin{array}{l}\text { Autoridades } \\
\text { competentes }\end{array}$ & \\
\hline & França & Ministério da Agricultura & $\begin{array}{l}\text { Departamento de } \\
\text { Serviços Veterinários do } \\
\text { Ministério da Agricultura }\end{array}$ & \\
\hline & Holanda & $\begin{array}{l}\text { Ministério da Saúde Pública, } \\
\text { Bem-estar e Esporte }\end{array}$ & $\begin{array}{l}\text { Inspetoria de Saúde } \\
\text { Publica Veterinária e } \\
\text { Proteção da Saúde }\end{array}$ & \\
\hline \multirow{3}{*}{ Europa } & Reino Unido & Home Office & $\begin{array}{c}\text { Grupo Ciência e } \\
\text { Pesquisa }\end{array}$ & $\begin{array}{l}\text { Divisão de } \\
\text { Procedimentos } \\
\text { Científicos em } \\
\text { Animais }\end{array}$ \\
\hline & Suécia & Ministério da Agricultura & $\begin{array}{l}\text { Autoridades designadas } \\
\text { pelo governo }\end{array}$ & $\begin{array}{l}\text { Autoridades } \\
\text { regionais e ou } \\
\text { municipais }\end{array}$ \\
\hline & Suíça & Conselho Federal & $\begin{array}{c}\text { Escritório Veterinário } \\
\text { Federal }\end{array}$ & $\begin{array}{c}\text { Autoridades } \\
\text { distritais do governo }\end{array}$ \\
\hline Oceania & Austrália & $\begin{array}{l}\text { Conselho Nacional de } \\
\text { Saúde e Pesquisa Médica }\end{array}$ & $\begin{array}{c}\text { Comitê de Bem-estar } \\
\text { Animal }\end{array}$ & \\
\hline
\end{tabular}

Africano de Pesquisa Médica (South African Medical Research CouncilMRC) (MRC, 2004), previstas pela Lei Sul Africana MRC (South African MRC Act) (1991), que por sua vez foi decretada pelo Presidente do Estado e pelo Parlamento (South Africa, 1991).

Animais são definidos nas diretrizes como vertebrados nãohumanos, incluindo embriões e fetos. CEUAs devem obrigatoriamente ser criadas em instituições de ensino e experimentação com animais, responsáveis pelo apoio dos pesquisadores na promoção do bemestar dos animais e auxílio na resolução de questões éticas relacionados com as pesquisas. Todos os projetos devem passar pela análise ética de uma CEUA indicada pelo MRC antes de serem iniciados. Tal CEUA deverá ser composta por no mínimo seis pessoas, incluindo médicos veterinários, pessoas com experiência em ensino e pesquisa com animais, especialistas em bemestar animal, pessoas sem experiência em experimentação, responsáveis pelo cuidado diário dos animais, além de pessoas treinadas em ética biomédica (MRC, 2004).

\section{Controle do uso de animais na América do Norte}

No Canadá, em 1963 o Conselho de Pesquisa Médica (Medical Research 
Council- MRC) requisitou que 0 Conselho Nacional de Pesquisa (National Research Council- NRC) estabelecesse uma comissão para investigar os cuidados e o uso de animais, culminando com a criação de um programa voluntário de controle exercido por cientistas em cada instituição, em 1968. O Conselho Canadense de Cuidados aos Animais (Canadian Council on Animal CareCCAC) foi estabelecido em 1982 objetivando a aplicação de princípios contidos no Guia para o Cuidado e Uso de Animais em Experimentação (Guide to the Care and Use of Experimental Animals). O uso de animais vertebrados e cefalópodes está sujeito aos requisitos do CCAC (CCAC, 2007).

As provisões legais canadenses referentes ao bem-estar animal, incluindo a proteção de animais usados para experimentos, estão presentes no Código Criminal (Criminal Code). Legislação específica tratando de animais em pesquisas, ensino e testes, pode ser observada em leis provinciais, como a Lei de Animais em Pesquisas (Animals for Research Act) de Ontário e Lei de Universidades (Universities Act) de Alberta (Olfert et al., 1993).

O CCAC é composto de 22 organizações-membro incluindo cientistas, educadores, médicos veterinários, representantes da indústria e de movimentos de proteção animal, que realizam visitas não-avisadas às instituições participantes, pelo menos a cada três anos. Os chamados painéis de avaliação são formados por pessoas com conhecimentos em várias áreas da experimentação, incluindo o bem-estar animal. Os participantes que obtêm sucesso na avaliação recebem um Certificado de Boas Práticas com Animais (Good Animal Practice - GAP). Uma comissão de cuidados aos animais é requerida em locais nos quais ocorre experimentação. As avaliações levam em conta o princípio dos Três Rs
(Russell e Burch, 1992) e documentos produzidos pelo CCAC (Olfert et al., 1993).

O CCAC recebe financiamento do Instituto Canadense de Pesquisa em Saúde (Canadian Institutes of Health Research- CIHR) e do Conselho de Ciências Naturais e Pesquisa em Engenharia (Natural Sciences and Engineering Research CouncilNSERC), além de departamentos federais de ciência básica e instituições privadas. As instituições são avaliadas através de grupos externos escolhidos pelo CIHR e pelo NSERC (CCAC, 2007).

Nos Estados Unidos, a Lei de Bem-estar Animal (Animal Welfare ActAWA) foi criada em 1966, determinando padrões mínimos de cuidados para animais criados para venda, pesquisas e transporte. Ela sofreu emendas em 1970, 1976, 1985, 1990 e 2002 (APHIS, 2005). Experimentos com animais são relatados desde 1973, sendo que registros de informações são mantidos somente sobre aves e mamíferos, com exceção de aves criadas para pesquisas, ratos e camundongos (Silverman al., 2007). Esta aberração exclui da proteção um grande contingente de animais de laboratório e faz com que os números relatados necessitem de análise crítica. Segundo Rollin (2006), tal lei é incoerente na tentativa de regulamentar o uso de animais para ensino e pesquisa e está longe de ser moralmente adequada.

O controle do uso de animais nos Estados Unidos é responsabilidade do Departamento de Agricultura do governo (United States Department of Agriculture-USDA) (USDA-APHIS, 2007), sendo que o Secretário da Agricultura tem a função de aplicar a AWA. Esta tarefa é delegada ao Serviço de Inspeção de Saúde de Animais e Plantas (Animal and Plant Health Inspection Service- APHIS) (APHIS, 2005). O APHIS determina e implanta 
padrões de cuidados para seres humanos e animais, mantém registros de números de animais usados e publica guias como o Manual de Regras para Cuidados aos Animais (Animal Care Policy Manual) de 2007, direcionado a inspetores do APHIS e proprietários (USDA-APHIS, 2007).

De acordo com Rudacille (2001), a AWA é aplicada pelo USDA/APHIS através de unidades de Execução Regulatória e Cuidados aos Animais (Regulatory Enforcement and Animal Care- REAC), as quais inspecionam laboratórios e compilam estatísticas relacionadas ao uso de animais. Segundo Shapiro (2000), a lei requer que indivíduos ou estabelecimentos de pesquisa sejam registrados pela APHIS e Oficiais Médicos Veterinários (Veterinary Medical Officers- VMOs) inspecionem tais locais.

Como parte do processo de regulamentação nos Estados Unidos, torna-se importante salientar a presença de Comitês Institucionais de Cuidados e Uso de Animais (Institutional Animal Care and Use Committees- IACUCs) (Silverman et al., 2007). Os IACUCs passaram a ser requeridos através da Lei de Extensão da Pesquisa em Saúde (Health Research Extension Act- HREA) e da Lei de Melhoria dos Padrões para Animais de Laboratório (Improved Standards for Laboratory Animals ActISLA), aprovadas em 1985. Uma declaração escrita após a avaliação de um projeto por um IACUC confirma que ele será conduzido sob a política do Serviço de Saúde Pública (Public Health Service- PHS), do Guia IACUC e da AWA (Shapiro, 2000). O trabalho de avaliação local e avaliação dos projetos deve sempre levar em conta o bemestar dos animais (USDA-APHIS, 2005).

\section{Controle do uso de animais na Ásia}

A regulamentação referente ao uso de animais de laboratório no Japão é de difícil compreensão para estrangeiros pela barreira criada pelo idioma. A maioria dos documentos é escrita em japonês e sua tradução formal não está disponível. Depois de uma revisão da legislação em 2005 e 2006, a tradução para o inglês de artigos relevantes está publicada como Diretrizes Fundamentais para a Conduta Apropriada de Experimentação Animal e Atividades Relacionadas em Instituições Acadêmicas de Pesquisa (Fundamental Guidelines for Proper Conduct of Animal Experiment and Related Activities in Academic Research Institutions) sob a jurisdição do Ministério da Educação, Cultura, Esportes, Ciência e Tecnologia (Ministry of Education, Culture, Sports, Science and Technology), como Ministério da Educação, Cultura, Esportes, Ciência e Tecnologia, Notificação No. 711 (Ministry of Education, Culture, Sports, Science and Technology, Notice No. 711) e Diretrizes para a Conduta Apropriada de Experimentação Animal pelo Conselho da Ciência do Japão (o Guia) (Guidelines for Proper Conduct of Animal Experiments by Science Council of Japan (the Guide)) (Kurosawa, 2007).

O sistema é organizado de forma hierárquica. A legislação superior é a Lei de Tratamento e Manejo Humanitário de Animais (The Law for the Humane Treatment and Management of Animals (Law No. 105, 1973)), revisada pela última vez em 2005. Tal lei determina que as pessoas devem evitar a morte desnecessária e injúrias aos animais, além de determinar a aplicação do conceito dos Três Rs (Russell e Burch, 1992) em experimentação (Kurosawa, 2007). O que difere a lei daquela de outros países é o fato de não ser obrigatório relatar o número de animais usados (Matsuda, 2004). Em segundo lugar estão as Normas, sendo que no caso dos animais de experimentação, aplica-se a Norma Relativa ao Cuidado, Manejo e Alívio da Dor e Distresse de Animais de Laboratório (Standard 
Relating to the Care, Management and Alleviation of Pain and Distress of Laboratory Animals (the Standard)) (Kurosawa, 2007).

Segundo Kurosawa (2007), em seguida são encontradas Diretrizes para a Conduta Apropriada de Animais de Experimentação pelo Conselho da Ciência do Japão (Guidelines for Proper Conduct of Animal Experiments by Science Council of Japan (the Guide)). Tal guia enfatiza a responsabilidade do diretor da instituição de pesquisa, estabelece a existência de CEUAs e recomenda que exista um sistema voluntário de controle de experimentação animal sob a responsabilidade dos pesquisadores, cuja intenção de publicação é o desenvolvimento de um guia para a conduta da experimentação animal, a exemplo do CCAC (CCAC, 2007). Finalmente existem regras determinadas pela instituição que realiza experimentação animal (Kurosawa, 2007).

Em universidades, todos os experimentos que utilizam animais são avaliados por CEUAs estabelecidas por ordem do Ministério da Educação, Cultura, Esportes, Ciência e Tecnologia, desde 1998 (Matsuda, 2004). De acordo com Kurosawa (2008 - Informe verbal), as CEUAs devem consistir de especialistas em animais de laboratório, em experimentação animal, entre outros profissionais. Porém, não é requerida a presença de especialistas em bem-estar animal nas comissões ou em qualquer parte do processo regulamentador.

\section{O controle do uso de animais na Europa}

Dois documentos legais referemse à competência das instituições pesquisadoras que utilizam animais na Europa. A Convenção Européia para a Proteção de Animais Vertebrados Usados em Experimentos e Outros Propósitos Científicos - ETS n9123
(European Convention for the Protection of Vertebrate Animals used for Experimental and other Scientific Purposes- ETS n923) (Council of Europe, 1986) objetiva a redução do número de experimentos e animais usados. Também determina que os países não devem realizar experimentos com animais, exceto se não houver alternativas, e que a pesquisa sobre métodos alternativos deve ser estimulada. Este documento foi organizado pelo Conselho da Europa, que envolve 47 países. A Diretiva 86/609 da União Européia com 27 países membros (Conselho das Comunidades Européias, 1986), além dos propósitos do documento anterior, requer que sejam adotados instrumentos legais implementando padrões de manutenção e cuidados com os animais, treinamento de pessoal e supervisão das atividades científicas (Paixão, 2007).

Segundo a Comissão das Comunidades Européias (2006), em 2006 foi estabelecido um Plano de Ação Comunitário relativo à Proteção e Bemestar Animal 2006-2010 para a Comunidade Européia, tendo em vista entre outras ações, a revisão da Diretiva $86 / 609$. Dentre os objetivos do plano estão definir a direção das políticas comunitárias em matéria de proteção e bem-estar dos animais, continuar a promoção de normas de bem-estar animal na União Européia e, internacionalmente, assegurar maior coordenação dos recursos existentes e apoiar o princípio dos Três Rs (Russell e Burch, 1992).

Analisando a legislação de alguns países europeus, observa-se a presença de particularidades no sistema regulamentador referente ao uso de animais em ensino e pesquisa.

A lei de proteção animal alemã (Animal Welfare Act-AWA) data de anos anteriores a 1934 e sua última revisão ocorreu em 2006. A AWA alemã protege 
animais vertebrados, invertebrados cefalópodes e decápodes, além de animais geneticamente modificados. Os experimentos somente podem ser realizados em vertebrados se a dor ou sofrimento a que os animais serão submetidos for eticamente justificada. É proibida a utilização de animais em testes com armas, tabaco, detergentes e cosméticos e o uso de animais em ensino somente é permitido no caso de não haver métodos alternativos (Seehofer, 2006).

O Ministério Federal de Alimentos, Agricultura e Proteção do Consumidor (Federal Ministry of Food, Agriculture and Consumer Protection) é o responsável pela aplicação da lei. O procedimento em caso de uso de animais em pesquisa ou ensino é submeter um projeto à autoridade competente, informando detalhes dos experimentos que serão realizados. As autoridades devem apontar comissões para auxiliá-los na decisão de autorizar as pesquisas, compostas de especialistas em medicina veterinária, medicina ou outra área de conhecimento necessária para realizar os experimentos, bem como de membros de organizações de bem-estar animal, estes representando um terço do total (Seehofer, 2006).

Todas as instituições de pesquisa apontam um Oficial de Bem-estar Animal interno para aconselhar o uso de animais, especialista nas áreas de medicina veterinária, medicina ou biologia. Estes garantem o bem-estar dos animais, aconselham a instituição na condução dos experimentos e trabalham para a redução das pesquisas em animais. A permissão para a realização de experimentos é concedida somente a pessoas com graduação em medicina veterinária, medicina, ciências naturais ou pessoas com qualificação suficiente. responsável pelas pesquisas deverá garantir que profissionais com competência suficiente estejam envolvidos nas atividades e que os experimentos sejam limitados ao mínimo possível. Registros de números de animais e procedimentos devem ser mantidos por pesquisadores e coordenadores das pesquisas, para posterior inspeção por parte das autoridades competentes (Seehofer, 2006).

A primeira legislação de proteção animal na França data de 1850. Entretanto, somente em 1963 a legislação passou a ser aplicada aos animais de experimentação. Em 1968, um decreto determinou que a experimentação animal deve ser realizada mediante a supervisão de uma pessoa portadora de autorização. Finalmente uma lei de 1976, relacionada à proteção da natureza, mudou o status dos animais e os reconheceu como seres sencientes (GIRCOR, 2007). Atualmente, um decreto de 1987 e três ordens ministeriais de 1988 mantêm as provisões das leis anteriores e incorporam a Diretiva Européia 86/609 (Conselho das Comunidades Européias, 1986). As regulamentações são aplicadas a animais vertebrados vivos e os experimentos somente podem ser realizados após a entrega de uma justificativa considerando o sofrimento envolvido (GIRCOR, 2007).

$\mathrm{Na}$ França, experimentação animal somente pode ser realizada sob a responsabilidade de um indivíduo munido de licença pessoal emitida pelo Ministério da Agricultura. Esta autorização especifica a área de atividade, a espécie animal utilizada e os protocolos de experimentação. Os proponentes da pesquisa devem ter um mínimo de educação sobre a biologia da espécie com a qual trabalham e treinamento dos procedimentos experimentais (GIRCOR, 2007).

As instalações nas quais os animais são mantidos também devem ser aprovadas pelo Ministério da 
Agricultura e por pessoas que detêm responsabilidade administrativa sobre a instituição que propõe o experimento. A aprovação inclui 0 tipo de experimentação, a espécie de animal usado, os protocolos a serem aplicados e a descrição estatística do número necessário de animais. Para manter animais selvagens, há necessidade de um certificado de instalação do Ministério do Meio Ambiente. Os animais devem ser obtidos de estabelecimentos certificados (GIRCOR, 2007).

A Comissão Nacional de Experimentação Animal (Commission Nationale de I 'Experimentation Animale), presidida por um conselheiro do estado, é encarregada de apresentar comentários ou elaborar propostas em todos os aspectos da presente legislação. Esta comissão é também consultada no que se refere ao treinamento e desenvolvimento de métodos alternativos ao uso de animais (GIRCOR, 2007).

$\mathrm{Na}$ Holanda, a legislação de 1977 determinava que o governo concedesse licenças aos chefes de instituições científicas com base na descrição da pesquisa a ser realizada, porém não havia controle externo de projetos. Em 1997 surgiu a Lei de Experimentos em Animais (The Dutch "Experiments on Animals Act'), determinando a proteção de animais vertebrados e espécies de invertebrados a serem designadas pelo governo. Experimentos permitidos incluem pesquisas de produtos biológicos, farmacológicas e toxicológicas, diagnóstico de doenças, propósitos educacionais e soluções para questões científicas. A experimentação não inclui desenvolvimento ou testes de cosméticos. Os animais usados devem ser criados em instituição portadora de licença, garantindo seu tratamento adequado, de acordo com a legislação (Ministry of Health, Welfare and Sport, 2001).
Toda pesquisa deve ser conduzida sob a licença do Ministério da Saúde, Bem-estar e Esporte (Ministry of Health, Welfare and Sport) e seu portador deverá apontar um médico veterinário ou outro especialista para supervisionar - bem-estar dos animais, manter registros de procedimentos e animais usados e os fornecer ao Ministério. Oficiais da Inspetoria de Saúde Publica Veterinária e Proteção da Saúde (Health Protection and Veterinary Public Health/ National Commodity Inspectorate) têm a obrigação de garantir o cumprimento da legislação e o poder de inspecionar os locais de pesquisa (Ministry of Health, Welfare and Sport, 2001).

O Ministério determina a criação de comissões de ética locais compostas por no mínimo sete membros representados por especialistas em experimentação animal, métodos alternativos, ética, bem-estar e proteção animal. Projetos que receberem recomendação negativa podem apelar à Comissão Central de Experimentação Animal, composta de cinco a nove membros, incluindo o presidente, especialistas em experimentação animal, animais de laboratório, bemestar animal, além do secretariado (Ministry of Health, Welfare and Sport, 2001).

No Reino Unido existe em caráter específico a Animal (Scientific Procedures) Act 1986 - ASPA (1986), que regulamenta 0 uso de animais em testes, pesquisas e ensino. A ASPA protege os animais vertebrados vivos, incluindo formas imaturas com mais de $50 \%$ do tempo de gestação ou incubação ou que tenham alcançado o estágio de vida livre. Mais tarde a lei passou a proteger também o cefalópode Octopus vulgaris. Ela protege animais usados para propósitos científicos que possam causar dor, sofrimento, distresse ou injúria duradoura. A maioria das espécies deve ser obtida de estabelecimentos criadores ou 
fornecedores possuindo certificado (Home Office, 2007).

A aplicação da ASPA está sob responsabilidade do Home Office (Home Office, 2007), um departamento governamental como o Ministério do Interior em outros países (Fry, 2008 Informe verbal). A Divisão de Procedimentos Científicos em Animais (Animals Scientific Procedures DivisionASPD), pertencente ao Grupo Ciência e Pesquisa (Science and Research Group) do Home Office, é responsável pelo controle. A ASPD divide-se em (1) seção administrativa, que emite licenças para pesquisas envolvendo animais e (2) Inspetoria de Procedimentos Científicos em Animais (Animals Scientific Procedures InspectorateASPI), responsável pela avaliação de projetos, por fazer recomendações quanto ao que deve ser licenciado, inspecionar o trabalho localmente $e$ difundir informações sobre boas práticas. Desde 1989, o Home Office tem editado Códigos e Práticas para alojamento e cuidados com animais em procedimentos científicos (Home Office, 2007). Tais códigos são desenvolvidos por meio de consultas à comunidade científica, organizações de criadores de animais de laboratórios e organizações de bem-estar animal (Fry, 2008 Informe verbal).

A lei britânica requer que pesquisadores que utilizam animais obtenham licenças pessoais, que 0 programa de trabalho envolvendo procedimentos científicos seja coberto por licenças para os projetos e que os estabelecimentos nos quais os procedimentos são conduzidos tenham um certificado (Home Office, 2007). Desta forma, o Reino Unido exerce controle separadamente em três níveis (Fry, 2008 - Informe verbal), o que representa um sistema mais refinado de controle.

Os Inspetores do Home Office podem fazer visitas não-avisadas aos estabelecimentos para verificar 0 trabalho, o cuidado e o alojamento dos animais. Porém, eles não têm poder de modificar ou suspender licenças e certificados, exceto para requerer que um animal em excessivo sofrimento sofra eutanásia humanitária, sendo obrigados a relatar estes fatos ao Home Office (Fry, 2008 - Informe verbal).

No Reino Unido a avaliação de propostas, que em outros países é feita por CEUAs, fica a cargo do ASPI. Apesar disso, os estabelecimentos também devem manter CEUAs locais para considerar a ética da pesquisa. Há ainda uma comissão nacional, a Comissão de Procedimentos em Animais (Animal Procedures Committee), composta de pesquisadores, veterinários, médicos, advogados, filósofos e especialistas em bem-estar animal, que aconselha 0 governo sobre problemas relativos a ASPA (Fry, 2008 - Informe verbal).

$\mathrm{Na}$ Suécia, a regulamentação referente aos animais usados em ensino e pesquisa é determinada pela Lei de Bem-estar Animal (The Animal Welfare Act) e pela Regulamentação de Bemestar Animal (The Animal Welfare Ordinance), ambas de 1988. Uma permissão para a realização de experimentos em animais é necessária em caso de utilização de mamíferos, aves, répteis, anfíbios, peixes e Cyclostomata, sendo que devem ser garantidos local adequado e cuidados necessários aos animais. As pesquisas somente devem ser realizadas se não existirem métodos alternativos e em caso de necessidade, o número mínimo de indivíduos deve ser envolvido, além de somente ser possível a utilização de animais especialmente criados para este propósito (Ministry of Agriculture, 2007).

O controle oficial relativo à aplicação da legislação é realizado pelo Ministério da agricultura, através de conselhos administrativos dos 
condados, pelas municipalidades e por outras autoridades designadas pelo governo, incluindo o Conselho de Agricultura e oficiais veterinários. Em nível municipal, o controle ocorre por meio de comitês que devem ter acesso a profissionais treinados em bem-estar animal, para que possam aplicar a legislação de maneira adequada (Ministry of Agriculture, 2007).

Os trabalhos envolvendo experimentação devem ser aprovados por uma comissão de ética. O número e os locais das comissões, o número de membros, bem como o presidente e vice-presidentes, são decididos pelo Conselho Sueco de Agricultura (Swedish Board of Agriculture). São compostas de presidente, vicepresidentes, pesquisadores e pessoas que trabalham com 0 manejo dos animais. Advogados compõem 50\% da comissão, sendo que os defensores dos direitos dos animais representam menos da metade desta classe. As licenças para os projetos têm validade de três anos. Em caso de aprovação da pesquisa, deverá haver um responsável por sua condução aprovado pelo governo, um médico veterinário dedicado à promoção da saúde dos animais e pessoal treinado para 0 manejo dos animais. Estes profissionais deverão assegurar o cumprimento da lei e relatar à autoridade as atividades realizadas e informações relativas aos animais usados (Ministry of Agriculture, 2007).

A regulamentação de atividades de ensino e pesquisa com animais na Suíça é baseada na Lei de Bem-estar Animal (Animal Welfare Act) de 1978 e nas Regulamentações de Proteção Animal (Animal Protection Ordinance) de 1981 e 1998. Algumas diretrizes legais regulam detalhes de licenciamento, requerimentos de manutenção, registros, etc. A legislação aplica-se a animais vertebrados, à ordem decápoda e à classe cefalópoda.
Os animais usados devem ser criados na instituição pesquisadora ou obtidos de criadores autorizados. Animais selvagens podem ser obtidos da natureza e domésticos podem ser usados em experimentos mesmo que não tenham sido criados com este propósito, com exceção de cães, gatos e coelhos (FVO, 2008).

O Conselho Federal (Federal Council) delega a aplicação da legislação ao Escritório Veterinário Federal (Federal Veterinary OfficeFVO), que por sua vez controla as autoridades regionais. A licença é concedida a pesquisadores graduados em biologia, medicina, medicina veterinária e algumas outras áreas, com três anos de experiência. Eles devem estar familiarizados com 0 tipo de animal, seu uso em experimentos, além de ser capazes de prover cuidados adequados. Através de diretrizes relacionadas aos princípios éticos na experimentação animal, o FVO declara que todas as pessoas envolvidas com pesquisas científicas devem passar por treinamento em bem-estar animal e apoiar o desenvolvimento de métodos alternativos (FVO, 2008).

Para obter uma licença para experimentação animal o pesquisador deve preencher um formulário fornecendo informações sobre o estudo, submetê-lo ao Escritório Veterinário Distrital, que toma as decisões com base na recomendação de uma comissão de especialistas em experimentação. Uma comissão semelhante também existe para aconselhar o FVO. Este requerimento é julgado a fim de se verificar se está dentro da legalidade e se os métodos são capazes de alcançar os objetivos esperados. A licença não é concedida se existirem métodos alternativos. Um relatório dos experimentos tem de ser produzido e mantido por no mínimo três anos (FVO, 2008). 


\section{Controle do uso de animais na Oceania}

Na Austrália, o Código de Práticas para Utilização e Cuidados de Animais Usados para Propósitos Científicos (Australian Code of Practice for the Care and Use of Animals for Scientific Purposes), editado em 1979 pelo Conselho Nacional de Saúde e Pesquisa Médica (National Health and Medical Research Council- NHMRC), objetiva aumentar os cuidados aos animais usados em pesquisas e orientar pesquisadores, professores, instituições e comissões de ética (Australian Government, 2004).

O uso de animais inclui pesquisas, ensino, testes em campo e de produtos, diagnósticos, fabricação de produtos biológicos e estudos ambientais (Australian Government, 2004). Todas estas atividades devem considerar 0 conceito dos Três Rs (Russell e Burch, 1992). Semelhante ao observado no Reino Unido (Home Office, 2007), a utilização em ensino e pesquisa inclui animais vertebrados não-humanos e invertebrados cefalópodes. Animais em fases iniciais de desenvolvimento, tais como estágio embrionário, fetal e formas larvais, com mais da metade do período de gestação ou incubação, ou quando podem se alimentar independentemente, são considerados capazes de sentir dor ou distresse (Australian Government, 2004).

O controle do uso de animais em ensino e pesquisa é responsabilidade dos governos Estaduais e Territoriais e o Código é a base do processo. Além do Código, o NHMRC disponibiliza guias, diretrizes e informações relacionadas à criação, cuidados e o uso de animais transgênicos, animais clonados, primatas não-humanos e cães usados em pesquisas, produção de anticorpos monoclonais, treinamento de médicos veterinários e informações para membros de comissões de ética. Também existe uma Comissão de Bem- estar Animal (The Animal Welfare Committe) pertencente ao NHMRC, que soluciona questões relativas ao bemestar e ética na experimentação (Australian Government, 2007).

As instituições pesquisadoras devem estabelecer uma ou mais CEUAs, as quais aprovam diretrizes para o cuidado dos animais, monitoram a aquisição, transporte, produção, acomodação, cuidado e uso dos animais, descrevem a forma de participação dos membros e examinam propostas de uso de animais. Cada CEUA é composta de no mínimo quatro pessoas, pertencentes a uma das categorias: qualificação em ciências veterinárias e com experiência em experimentação; experiência em uso de animais para ensino e pesquisa; comprometida com o bem-estar animal, não-vinculada à instituição; nãovinculada à instituição e que nunca tenha se envolvido em atividades de ensino e pesquisa com animais (Australian Government, 2004).

\section{Controle do uso de animais no Brasil}

Uma análise histórica do controle do uso de animais no Brasil revela que algumas leis buscam atender à questão da prevenção do sofrimento animal. A primeira legislação surgiu em 1924, através do Decreto no 16.590, regulamentando atividades relativas à diversão que causassem sofrimento aos animais (Maschio, 2002). Em 1934 surgiu o Decreto no 24.645. Seu conteúdo é abrangente, considerando em sua maior parte animais de grande porte. Ele determina que os animais existentes no país são tutelados do estado e traz alíneas sobre maus-tratos e o uso de animais para experimentos científicos e ensino (Brasil, 1934). Em 1968 cria-se a Lei Federal no 5.517, que dispõe sobre o exercício da profissão de Médico Veterinário e cria os Conselhos Federal e Regionais de Medicina Veterinária. O capítulo II trata da 
competência privativa dos médicos veterinários nas atividades e locais nos quais ocorra pesquisa com animais (Brasil, 1968). E lei é regulamentada pelo Decreto № 64.704 de 1969 (Brasil, 1969).

Em 1979 surgiu a primeira tentativa de se estabelecer normas para a vivissecção didático-científica em animais, através da Lei $n^{\circ}$ 6.638. Tal lei determina que biotérios e centros de experiências com animais devem ser registrados em órgão competente e por ele autorizados a funcionar (Brasil, 1979). Em 1991, a Sociedade Brasileira de Ciência em Animais de Laboratório (SBCAL-COBEA) publicou os Princípios Éticos na Experimentação Animal. $\mathrm{Na}$ falta de regulamentação específica, este texto passou freqüentemente a nortear a conduta de pesquisadores e professores. A sociedade foi fundada em 1983 sob a denominação de Colégio Brasileiro de Experimentação Animal (COBEA) e seu objetivo principal é programar e estimular pesquisas no campo da experimentação animal (SBCAL-COBEA, 2009); desta forma, tal sociedade não deve agir como órgão regulamentador, uma vez que fomenta os experimentos com animais.

Outra medida importante foi a Resolução $n^{\circ} 592$ de 1992 criada pelo Conselho Federal de Medicina Veterinária (CFMV, 1992), que gerou preceitos legais determinando entre outras providências, o registro obrigatório de biotérios nos Conselhos Federal e Regionais de Medicina Veterinária.

No que se refere à criação e manutenção da fauna silvestre brasileira para experimentação, a partir da Portaria no 16 de 1994 do Instituto Brasileiro do Meio Ambiente e dos Recursos Naturais Renováveis- IBAMA, as universidades e centros de pesquisa devem ser registrados junto a este órgão. Além disso, os pesquisadores têm de manter um sistema de controle de fuga dos animais, prestar informações sobre o local, firmar termo de compromisso assegurando a manutenção dos animais e encaminhar ao IBAMA cópia de trabalhos publicados decorrentes da pesquisa. Ao final da pesquisa os animais podem ser transferidos para instituições afins, o que possibilita sua reutilização (IBAMA, 1994).

Em 1998 o governo federal aprovou a Lei de Crimes Ambientais, $\mathrm{n}^{\circ}$ 9.605, na qual considera crime "praticar ato de abuso, maus-tratos, ferir ou mutilar animais silvestres, domésticos ou domesticados, nativos ou exóticos, além de realizar experiências dolorosas ou cruéis em animal vivo, ainda que para fins didáticos ou científicos, quando existirem alternativas". A lei prevê penalidades para o uso indiscriminado de animais, mas não estabelece órgão fiscalizador das instituições que praticam a vivissecção (Brasil, 1998). A regulamentação desta lei se deu através do Decreto no 6.514 de 2008 (Brasil, 2008a). Segundo Levai (2004), ela uniformizou a situação dos animais, criminalizando a conduta daqueles que atentarem contra os animais que estejam em território brasileiro.

Em 2007 foi apresentado à Câmara Federal o PL no 215, de autoria do Deputado Ricardo Trípoli, instituindo de forma prescritiva o Código Federal de Bem-estar Animal. O objetivo é chegar a um modelo adequado para o bem-estar animal, avançando no campo da experimentação (Trípoli, 2007). O projeto contém um livro tratando do uso de animais em experimentação, funcionamento, composição e atribuições de Comissões de Ética e Bem-estar em Experimentação, criação, manejo e destinação dos animais, bem como da objeção de consciência (Brasil, 2007).

Em 1995 criou-se o Projeto de Lei (PL) no 1.153/1995, de autoria do Deputado Sérgio Arouca, tratando da 
criação e do uso de animais para atividades de ensino e pesquisa. Em 1997 este projeto foi apensado e passou a constituir o PL $\mathrm{n}^{-}$3.964. Dentre os pontos importantes previstos pelo PL $\mathrm{n}^{\circ}$ 3.964/1997 destaca-se a criação de um órgão controlador das atividades de ensino e de pesquisa com animais, a criação de CEUAs nas instituições que pratiquem a experimentação e a definição de penalidades às instituições e profissionais pelo emprego indevido das normas. Em 2003, foi criado o PL no 1.691 que dispõe sobre o uso de animais para fins científicos e didáticos e estabelece a escusa de consciência à experimentação animal, sendo também apensado ao PL no $1.153 / 1995$ (Cardoso, 2003). Em outubro de 2008 foi aprovada a Lei Federal $n \circ 11.794$ (Brasil, 2008b), também conhecida como Lei Arouca, que cria o Conselho Nacional de Controle da Experimentação Animal (CONCEA), dentro do Ministério da Ciência e Tecnologia. Em julho de 2009, foi aprovado o Decreto no 6.899 (Brasil, 2009) que regulamenta a referida lei.

Atualmente no Brasil os animais de laboratório estão protegidos em nível federal principalmente pela Lei no 9.605 (Brasil, 1998). Adicionalmente, em 2008 - Conselho Federal de Medicina Veterinária instituiu a Resolução nํ 879, que dispõe sobre a normatização do uso de animais sencientes em ensino e pesquisa em Medicina Veterinária e Zootecnia, além da criação de CEUAs nas instituições de ensino superior e de pesquisa nestas áreas (CFMV, 2008). Ainda, podem ser citados a Lei Federal no 11.794 (Brasil, 2008b) e o Decreto no 6.899 (Brasil, 2009).

\section{O controle do uso de animais no Paraná}

Alguns estados brasileiros promulgaram leis de proteção animal que tratam da vivissecção, como a Lei no 11.977 de 2005 do estado de São
Paulo, a Lei n`3.900 de 2002 do estado do Rio de Janeiro e a Lei $n^{\circ} 11.915$ de 2003 do estado do Rio Grande do Sul. O Código Estadual de Proteção aos Animais do estado do Paraná (Lei no 14.037, de 2003) estabelece normas para a proteção dos animais no estado e, no capítulo que trata de animais de laboratório, determina que os centros de pesquisa sejam registrados no órgão competente e supervisionados por profissionais de nível superior. Antes de iniciar o projeto, o diretor da instituição deverá relatar ao órgão competente a natureza do experimento, a quantidade e a espécie dos animais usados e o nível de dor que os mesmos sofrerão. É proibida a prática de vivissecção sem uso de anestésico, sua realização em escolas de ensino fundamental e médio, bem como a importação ou exportação de animais para pesquisas (Paraná, 2003). Seus itens estão vigentes, a não ser pelo artigo 20 que determina que "o diretor do centro de pesquisa, antes de proceder a qualquer experimento com animal vivo, deverá relatar ao órgão competente a natureza do experimento, a quantidade e a espécie dos animais utilizados e o nível de dor que os mesmos sofrerão", pois o órgão competente não foi regulamentado.

No Paraná já existem iniciativas objetivando conhecer a quantidade de animais utilizados em pesquisa, demonstrando um início de organização de tais informações e esforços para a adequação à legislação vigente. Um estudo dos artigos científicos publicados durante o ano de 2006 em 19 periódicos paranaenses demonstrou que um número expressivo de animais vertebrados, ou seja, de alta probabilidade de senciência é utilizado no Estado do Paraná. O estudo utilizouse do método da amostragem bibliográfica, que apesar de apresentar algumas limitações, mostrou que 0 Brasil é importante em termos de 
números de animais usados em pesquisas (Silla et al., 2008).

\section{CONCLUSÃO}

Avaliando as informações obtidas nos diversos países, percebe-se uma grande variação na forma de regulamentação do uso de animais para ensino e pesquisa. Além dos movimentos sociais atuantes em prol da proteção animal, destaca-se o trabalho das comissões de ética e também a legislação específica em cada um dos países pesquisados. Em todos os países estudados, exceto o Brasil, verifica-se o controle do uso de animais com investimento de verba pública. Profissionais com conhecimento em bem-estar animal participantes do sistema regulamentador podem ser observados em dez países, representando $83 \%$ dos países estudados. Para a maioria dos países, o nível de regulamentação mais aplicado é a ação de CEUAs institucionais. As experiências internacionais podem servir como alicerce para o progresso do controle das atividades que utilizem animais para ensino e pesquisa no Brasil, em nível estadual e federal. A sociedade brasileira apresenta preocupação crescente com o tema e sua pronta abordagem trará benefícios em termos de aprimoramento ético do ambiente acadêmico e de pesquisa envolvendo animais.

\section{AGRADECIMENTO}

Os autores gostariam de agradecer ao senhor Derek J. Fry, Inspetor do Animals (Scientific Procedures) Inspectorate- Home Office, Londres, Inglaterra- durante os anos 1990 a 2002 e Inspetor chefe da mesma instituição de 2003 a 2007, pela sua especial contribuição para a presente revisão na seção referente à regulamentação do uso de animais em ensino e pesquisa no Reino Unido e à agência CAPES de apoio à pesquisa.

\section{REFERÊNCIAS}

ACEME, N. Animal Research Ethics in Africa: an overview. Acta Tropical, v.112, supplement 1, p.48-52, 2009.

ANIMAL (SCIENTIFIC PROCEDURES) ACT 1986. Disponível em: http://www.archive.officialdocuments.co.uk/document/hoc/321/321-xa.htm. Acesso em: 24/11/2007.

ANIMAL AND PLANT HEALTH INSPECTION APHIS, The animal care program and the U.S.

Department of Agriculture's authority under the animal welfare act: basic questions and answers, 2005. Disponível em:

http://www.aphis.usda.gov/lpa/pubs/fsheet_faq notice/faq_awusda.html. Acesso em: 25/07/2008.

AUSTRALIAN GOVERNMENT, Nacional Health and Medical Research Council. Animal research ethics. Disponível em:

http://www.nhmrc.gov.au/health_ethics/animal/in dex.htm. Acesso em: 03/11/2007.

AUSTRALIAN GOVERNMENT, Nacional Health and Medical Research Council. Australian code of practice for the care and use of animals for scientific purposes, 2004.Disponível em: http://www.nhmrc.gov.au/publications/synopses/ ea16syn.htm. Acesso em: 08/05/2008.

AUTOR DESCONHECIDO. Diretório de

Pesquisa: Comitês de ética em pesquisa animal no Brasil. Ciência e Cultura, v.60, n.2, p. 50-53, 2008.

BENSON, G.J.; ROLLIN, B.E. The Well-being of Farm Animals: challenges and solutions. lowa: Blackwell Publishing, 2004. 378 p.

BRASIL a, Decreto o 6.514 , de 22 de Julho de 2008. Disponível em:

http://www.planalto.gov.br/ccivil_03/_Ato20072010/2008/Decreto/D6514.htm\#art153. Acesso em: 07/08/2009.

BRASIL b, Lei oㅜ 11.794, de 8 de Outubro de 2008. Disponível em:

https://www.in.gov.br/imprensa/visualiza/index.js p?jornal $=1$ \&pagina $=1$ \&data $=09 / 10 / 2008$. Acesso em: 10/10/2008.

BRASIL, Decreto oㅜ 24.645 de 1934. Disponível em:

http://www6.senado.gov.br/legislacao/ListaPublic acoes . action?id=39567. Acesso em: 10/10/2007.

BRASIL, Decreto № 6.899 , de 15 the Julho de 2009. Disponível em: 
http://www.planalto.gov.br/ccivil_03/_Ato20072010/2009/Decreto/D6899.htm. Acesso em: 04/08/2009.

BRASIL, Projeto de lei no 215, de 2007.

Disponível em:

http://www2.camara.gov.br/proposicoes. Acesso em: 12/05/2008.

CANADIAN COUNCIL ON ANIMAL CARE CCAC, About CCAC. Disponível em:

http://www.ccac.ca/en/About_CCAC/About_CCA C_Main.htm. Acesso em 20/12/2007.

CARDOSO, C.V.P., Programa de Ação para Biotérios: leis referentes à experimentação animal no Brasil. Centro de Gestão e Estudos Estratégicos (CGEE), 2003. 12 p. Disponível em:

www.cgee.org.br/atividades/redirect.php?idProd uto=1688. Acesso em: 07/08//2009.

CHAVES, C.C. Relatório da disciplina de estágio supervisionado. 2000. Niterói, 45f. Relatório de Conclusão de Curso - Centro de Ciências Médicas, Universidade Federal Fluminense.

COMISSÃO DAS COMUNIDADES

EUROPÉIAS, Comunicação da comissão ao parlamento europeu e ao conselho: sobre um plano de ação comunitário relativo à protecção e ao bem-estar dos animais 20062010. Bruxelas, 2006. Disponível em: http://ec.europa.eu/food/animal/welfare/com_acti on_plan230106_pt.pdf. Acesso em: 25/04/2008.

CONSELHO DAS COMUNIDADES

EUROPÉIAS, European directive 86/609/CEE, 1986. Disponível em:

http://eurlex.europa.eu/smartapi/cgi/sga_doc?s martapi!celexapi!prod!CELEXnumdoc\&numdoc= $31986 \mathrm{~L} 0609 \&$ model $=$ guichett $\& \mathrm{lg}=$ pt. Acesso em: 24/11/2007.

CONSELHO FEDERAL DE MEDICINA

VETERINÁRIA - CFMV, Resolução nํ592, de 26 de junho de 1992. Disponível em:

http://www.cfmv.org.br/portal/legislacao/resoluco es/resolucao_592.htm. Acesso em: 08/05/2008.

CONSELHO FEDERAL DE MEDICINA VETERINÁRIA - CFMV, Resolução ํㅜ 879, de 15 de fevereiro de 2008. Disponível em: http://www.cfmv.org.br/portal/legislacao/resoluco es/resolucao_879.pdf. Acesso em: 08/05/2008.

COUNCIL OF EUROPE - Coe, European convention for the protection of vertebrate animals used for experimental and other scientific purposes (ETS n9123). Strasbourg, 1986. Disponível em:

http://conventions.coe.int/Treaty/en/Treaties/Htm 1/123.htm. Acesso em: 20/03/2008.
DIAS, E.C. A tutela jurídica dos animais. Belo Horizonte: Mandamentos, 2000. 421 p.

FEDERAL VETERINARY OFFICE - FVO,

Animal Welfare. Disponível em:

http://www.bvet.admin.ch/themen/tierschutz/inde x.html?lang=en. Acesso em: 22/04/2008.

FRY, D.J. Informe verbal. Informações relativas à forma de trabalho do Home Office. Mensagem recebida em Londres em: 17/07/2008.

FUND FOR THE REPLACEMENT OF ANIMALS IN MEDICAL EXPERIMENTS - FRAME, Laws and provisions in other countries. Disponível em: http://www.frame.org.uk/page.php?pg_id=154. Acesso em: 06/05/2008.

GREIF S., TRÉZ, T. A Verdadeira Face da Experimentação Animal: a sua saúde em perigo. Rio de Janeiro: Sociedade Educacional Fala Bicho, 2000. 200 p.

GROUPE INTERPROFESSIONEL DE REFLEXION ET DE COMMUNICATION SUL LA RECHERCHE - GIRCOR, French animal protection legislation and animal research. Disponível em:

http://www.ebra.org/france+1_14.htm. Acesso em: 15/09/2007.

HOME OFFICE, Scientific Procedures on Living Animals. Disponível em:

http://www.homeoffice.gov.uk/rds/scientific1.html Acesso em: 20/03/2008.

INSTITUTO BRASILEIRO DO MEIO AMBIENTE E DOS RECURSOS NATURAIS RENOVÁVEIS - IBAMA, Portaria no 016, de 04 de março de 1994. Disponível em: http://www.ibama.gov.br/fauna/legislacao/port_1 6_94.pdf. Acesso em: 07/05/2008.

KUROSAWA, T.M. Informe verbal. Informações sobre o funcionamento das CEUAs no Japão. Mensagem recebida por e-mail em: 09/08/2008.

KUROSAWA, T.M. Japanese regulation of laboratory animal care with $3 R$ s. In: WORLD CONGRESS ON ALTERNATIVES AND ANIMAL USE IN THE LIFE SCIENCES, 6., 2007, Tokyo. Proceedings... Tokyo: AATEX, 2007. p.317321.

LEVAI, L.F. Direito dos animais. Campos do Jordão: Mantiqueira, 2004. 159 p.

MASCHIO, J.J. Animais. Direitos deles e ética para com eles, 2002. Disponível em:

http://jus2.uol.com.br/doutrina/texto.asp?id=7142 . Acesso em: 21/07/2008.

MATSUDA, Y. Recent trends in the number of laboratory animals used in Japan. In: WORLD CONGRESS ON ALTERNATIVES AND ANIMAL USE IN THE LIFE SCIENCES, 4., 2002, New 
Orleans. Proceedings... New Orleans: ATLA, 2004. p.299-301.

MIDDLETON, D. The Animal Ethics Committee: getting it all to work. In: AUSTRALIAN AND NEW ZEALAND COUNCIL FOR THE CARE OF ANIMALS IN RESEARCH AND TEACHINGANZCCART, 2005, Wellington.

Proceedings...Wellington: The Royal Society of New Zealand, 2005. p.23-25.

MINISTRY OF AGRICULTURE, The animal welfare act \& the animal welfare ordinance. Stockholm, 2007. Disponível em: http://www.regeringen.se/content/1/c6/09/03/08/ 6793d541.pdf. Acesso em: 11/07/2008.

MINISTRY OF HEALTH, WELFARE AND SPORT, Animal experimentation in the Netherlands, 2001. Disponível em: http://www.vet.uu.nl/nca/userfiles/other/leaflet_a nimal_experimentation_in_the_Netherlands.pdf. Acesso em: 18/07/2008.

NACONECY, C.M. Sobre uma Ética da Vida: o biocentrismo moral e a noção de bio-respeito em ética ambiental. 2007. Porto Alegre, $142 f$. Dissertação (Doutorado em Filosofia) -

Programa de Pós-graduação em Filosofia, Pontifícia Universidade Católica do Rio Grande do Sul.

OLFERT, E.D. et al., Guide to the care and use of experimental animals, 1993. Disponível em: http://www.ccac.ca/en/CCAC_Programs/Guidelin es_Policies/GUIDES/ENGLISH/toc_v1.htm. Acesso em: 20/07/2008.

PAIXÃO, R.L. A experimentação animal em debate. Revista do Conselho Federal de Medicina Veterinária, jan./fev./mar./abr, n.40, p.59-66, 2007.

PAIXÃO, R.L. As comissões de ética no uso de animais. Revista do Conselho Federal de Medicina Veterinária, mai./jun./jul./ago, n.32, p.13-20, 2004.

PAIXÃO, R.L.; SCHRAMM, F.R.

Experimentação Animal: razões e emoções para uma ética. Niterói: EdUFF- Editora da Universidade Federal Fluminense, 2008. 206 p.

PALÁCIO DO PLANALTO, Decreto № 64.704, de 1969. Disponível em:

http://www.planalto.gov.br/ccivil_03/decreto/Anti gos/D64704.htm. Acesso em: 07/05/2008.

PALÁCIO DO PLANALTO, Lei Federal $n^{\circ} 6.638$, de maio de 1979. Disponível em:

http://www.planalto.gov.br/ccivil_03/Leis/19701979/L6638.htm. Acesso em: 10/10/2007.

PALÁCIO DO PLANALTO, Lei Federal n`9.605, de 12 de fevereiro de 1998. Disponível em:
http://www.planalto.gov.br/ccivil_03/Leis/L9605.h tm. Acesso em: 10/10/2007.

PALÁCIO DO PLANALTO, Lei Federal № 5.517, de 1968. Disponível em:

http://www.planalto.gov.br/ccivil_03/Leis/L5517.h tm. Acesso em: 10/10/2007.

PARANÁ, Código estadual de proteção aos animais- lei no 14.037, de 2003. Disponível em: http://celepar7cta.pr.gov.br/SEEG/sumulas.nsf/6 c0580efa19ff3ac83256fdd0065f99c/48e67ef7b8 1a182803256e9900690deb?OpenDocument. Acesso em: 31/01/2007.

RAYMUNDO, M.M.; GOLDIM, J.R. Ética da pesquisa em modelos animais. Bioética, v.10, n.1, p.31-44, 2002.

RODRIGUES, D.T. O direito e os Animais: uma abordagem ética, filosófica e normativa. Curitiba: Juruá, 2006. 164 p.

ROLLIN, B.E. Farm animal welfare: social, bioethical, and research issues. lowa: lowa State Press, 2003. 169 p.

ROLLIN, B.E. The Regulation of Animal Research and the Emergence of Animal Ethics: a conceptual history. Theoretical Medicine and Bioethics, v.27, n.4, p.285-304, 2006.

RUDACILLE, D. The Scalpel and the Butterfly: the conflict between animal research and animal protection. Berkeley: University of Califórnia Press, 2001. 390 p.

RUSSELL, W.M.S.; BURCH, R.L. The principles of humane experimental technique. London: Hiperion Books, 1992. Diponível em:

http://altweb.jhsph.edu/publications/humane_exp /het-toc.htm. Acesso em: 09/06/2008.

SEEHOFER, H. Publication of the Revision of the Animal Welfare Act of 18 May 2006. Federal Law Gazette, part I, n.25, p.1206-1222, 2006.

SHAPIRO, L.S. Applied animal ethics. United States of America: Delmar- Thomson Learning, 2000. $576 \mathrm{p}$.

SILLA, V.C.B. et al. Uso de animais para pesquisa de acordo com amostragem bibliográfica em periódicos científicos publicados no estado do paraná no ano de 2006. In: CONGRESSO BRASILEIRO DE BIOÉTICA E BEM-ESTAR ANIMAL, 1., 2008, Recife. Anais... Recife: Conselho Federal de Medicina Veterinária, 2008, Publicação em CD (resumo).

SILVERMAN, J. et al. The IACUC handbook. Boca Raton: CRC Press, 2007. 652 p.

SOCIEDADE BRASILEIRA DE CIÊNCIA EM ANIMAIS DE LABORATÓRIO - SBCAL-COBEA, disponível em: 
http://www.cobea.org.br/index.php?p=historico . Acesso em: 07/08/2009.

SOUTH AFRICA, South African Medical Research Council Act 1991. Disponível em: http://www.mrc.ac.za/about/MRCAct.pdf. Acesso em: 05/08/2009.

SOUTH AFRICAN MEDICAL RESEARCH COUNCIL - MRC, Guidelines on Ethics for Medical Research. Book 3: use of animals in research and training. Diponível em: http://www.sahealthinfo.org/ethics/index.htm . Acesso em: 05/08/2009.

THE ROYAL SOCIETY FOR THE PREVENTION OF CRUELTY TO ANIMALS RSPCA, About RSPCA: history. Disponível em: http://www.rspca.org.uk/servlet/Satellite?pagena me=RSPCA/RSPCARedirect\&pg=about_the_rsp ca\&marker $=1$ \&articleld $=996827934749$. Acesso em: 18/03/2008.

TRÍPOLI, R. Meu compromisso é com a vida. $\mathbf{0}$ Observador da Legislação Animal, ed.2, 2007. Disponível em:

http://www.wspabrasil.org/newsletter/maio2007/newsletter-maio-

2007.asp?nid=12\&type=iview. Acesso em: 24/11/2007.
UNITED STATES DEPARTMENT OF AGRICULTURE- ANIMAL AND PLANT HEALTH INSPECTION - USDA-APHIS, Animal welfare. Disponível em: http://www.aphis.usda.gov/. Acesso em: 05/05/2007.

UNITED STATES DEPARTMENT OF AGRICULTURE- ANIMAL AND PLANT HEALTH INSPECTION - USDA-APHIS, Animal Welfare Regulations: Title 9- animals and animal products. Subpart C- research facilities. Institutional animal care and use committee (IACUC), 2005. Disponível em: http://www.aphis.usda.gov/animal_welfare/downl oads/awr/9cfr2.31.txt. Acesso em: 30/07/2008. 